\title{
Design and implementation of a feedback programmable spin coating system
}

\author{
Ahmed K. Kadhim, Salah M. Swadi, Ghusoon M. Ali \\ Electrical Engineering Department, College of Engineering, Mustansiriyah University, Iraq
}

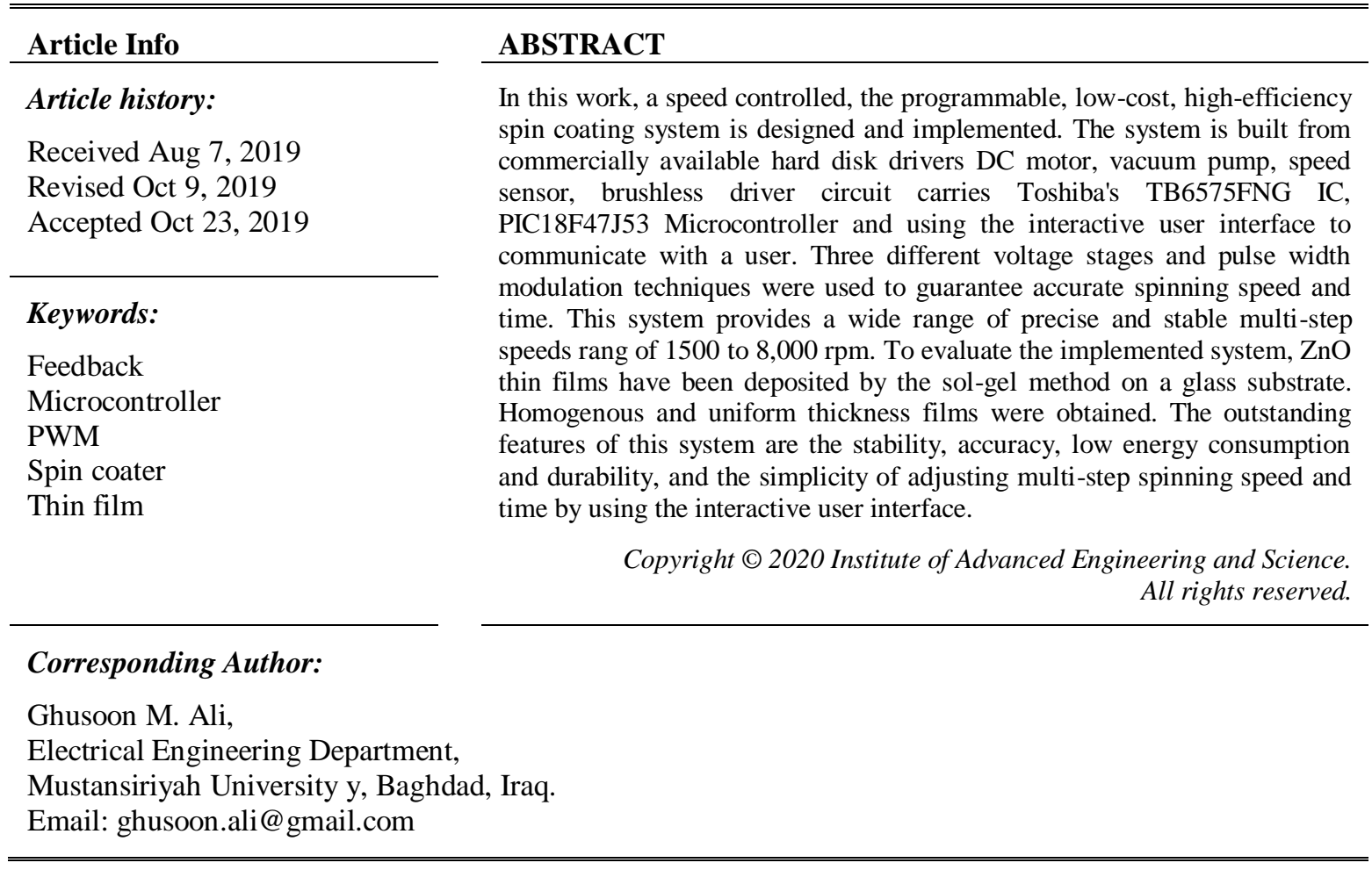

\section{INTRODUCTION}

Thin film deposition technique plays a key role in the electronic and optical applications manufacturing. Various techniques have been used to deposit thin films, including sputtering [1], chemical vapor deposition [2], thermal evaporation [3], spray pyrolysis [4], dip-coating [5, 6], electro-deposition [7] and spin coating [8]. As compared to other deposition techniques, spin coating is faster, easier, cheaper and need a small amount of depositing material [9]. In this technique, the thin films are deposited by employing centrifugal force concept, by adopting on speed-time changes [10, 11]. The thickness of a film is a result of a balancing of two main opposing forces namely: centrifugal force and viscosity force. The thin film thickness is significantly depending on spinning speed, solution viscosity and substrate type. During the rotation process, the centrifugal force overcomes gravity and produces rapid thinning of the film $[10,11]$.

There are many commercial spin coating systems available, however, many researchers have been and still proposed to build spin coater for research purposes [10, 12-14]. Most of the spin coater based research purposes work utilized brushless DC motor (BLDC motor), that usually employed as a computer hard drive using pulse width modulation (PWM) to control DC motor speed. PWM is a recognizable technique used to precise control DC motor speed by a controller circuit [15]. The speed is controlled by the average amount of power fed to the motor. By controlling the ON conduction time duration per cycle, the amount of voltage applied to the motor can be controlled. Changing the working cycle is employed through the built-in Module in the controller circuit [16]. In addition, the feedback control system is essential to be deployed to get accurate spinning speed [14]. To solve the degradation of spinning speed over longterm utilization. Also, to confirm the accurate spinning speed, regardless of the shape, size and mass 
substrates, within the limit of the system capabilities. However, most of the previously reported works are quite simple without the feedback circuit and using a single stage voltage [10, 12-14].

In this work, a developed programmable feedback spin coating system has been designed and implemented for an actual need of our research laboratory. The system is low cost, efficient and durable, low energy consumption and easy to operate. The system is built from a commercially available DC motor in HDD, vacuum pump, $12 \mathrm{~V}$ power supply, IR sensor, brushless click carries Toshiba's TB6575FNG IC and PIC18F47J53 Microcontroller, as well as to the external system body. The system is connected to the computer by Human interface device (HID) protocol for bi-directional communication, a serial transfer technology was used via the USB port. Three different voltage stages have been applied to obtain a smooth and wide variable range of spinning speed. An Infrared (IR) circuit was employed to sense the real-time precise spin speed. PIC18F47J53 microcontroller program was written using MikroC language which includes a variety of libraries to support the chip modules. The performance of the system was evaluated by coating $\mathrm{ZnO}$ sol-gel on glass substrates. The relation of the thickness of the films with the spinning speed and spinning time were investigated. Thin, consistent, controlled films was obtained.

\section{RESEARCH METHOD}

We utilized a commercially Brushless DC Motor (BLDC motor), as shown in Figure 1(a), that usually employed as a computer hard drive. This motor is proposed because it can be precisely controlled with a very long operating life, small dimensions, and high acceleration [10]. To hold the substrate, the suckering process is implemented. Thus, the BLDC rotor axis was hollowed out with a high-speed steel drill with a diameter of $5 \mathrm{~mm}$. A special nozzle is attached to the lower side of the hollow rotor axis through a hose and connects to the outlet of the vacuum pump. To ensure there is no leakage could be exists at the contact area between the nozzle and lower face of the dc motor, a special arrangement (consist of O-ring and spring thrust on the nozzle) is used. This arrangement allows the motor rotor to rotate freely and at the same time it's providing enough value of vacuum pressure to hold specimen on the DC motor dram. The vacuum pump model $1.5 \mathrm{CFM} \mathrm{VH} 115 \mathrm{~N}$ is chosen based on the friction force calculations. The motor torque is sufficient to fix the lightweight substrates ( $\mathrm{Si}$, glass, sapphire) for the required spinning speed range (1500 to $8000 \mathrm{rpm}$ ). The substrate holds on the top of the BLDC rotor drum during the coating process. This fixing method is preferably more than the adhesive material method, to avoid any damage could happen to the specimen after complete coating.

The PWM control technique is used to accurately control the spinning speed of the motor. PWM is done by sending different duty cycle (the variation in the ON state to OFF) with a fixed voltage. The device spinning speed relies on the supplied average power which has been formed as a wave of pulses (PWM) duty cycle. The supplied average power enforce the motor coils to produce a different torque and then different speed. Hence, the driver controls the rotation speed by changing the PWM duty cycle (D). For example, if the voltage reference of the driver is $10 \mathrm{~V}$ then the supplied voltage $(\mathrm{V}=(10 / 100) \times \mathrm{D})$ [14]. For the maximum value of designed spinning speed $(8000 \mathrm{rpm})$, the obtained spinning speed $(\omega=(8000 / 10) \times \mathrm{V})$. Hence, the relation of speed as a function of the duty cycle can describe, $\omega=(8000 / 100) \times D)$. If the duty cycle $100 \%$ gives the maximum speed.

\subsection{Control Circuit}

The controller circuit is built with a Microchip PIC18F47J53 microcontroller. The PIC18F47J53 microcontroller chip is one of the $18 \mathrm{~F}$ family 8 -bit architecture. This chip has different built-in modules. Table 1 listed the different modules used in this work to achieve the system implementation requirement. Figure 1(b) shows an image of the PIC18F47J53 microcontroller chip. Since the microcontroller output power is very small, a brushless motor driver called Brushless Click is utilized to enable the microcontroller to drive the 3-phase full-wave BLDC motor. The Click board manufactured by Mikroelektronika Company contains a TB6575FNG chip. The TB6575FNG chip is a three-phase PWM controller for sensorless BLDC motors. Figure 1(c) shows an image of the utilized Brushless Click.

Table 1. Modules used in the Project

\begin{tabular}{|c|c|}
\hline Module Name & Function in the project \\
\hline PWM & Controlling the BLDC \\
\hline GPIO & Emergency switch, Indication LEDs \\
\hline Timers & $\begin{array}{l}\text { Calculating time and counting some external } \\
\text { signal to extract motor speed }\end{array}$ \\
\hline USB & $\begin{array}{l}\text { To establish a connection between the } \\
\text { microcontroller and the PC }\end{array}$ \\
\hline
\end{tabular}




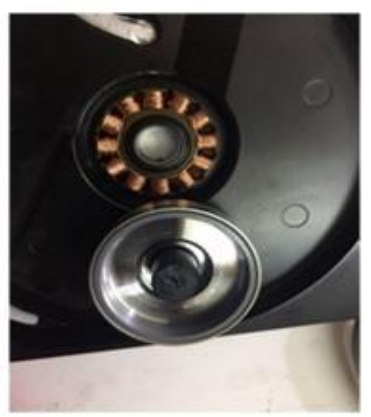

(a)

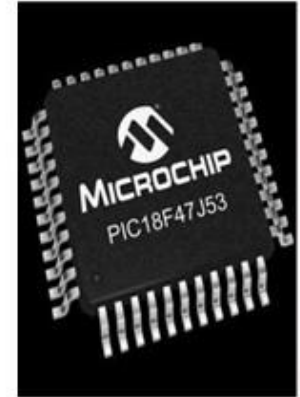

(b)

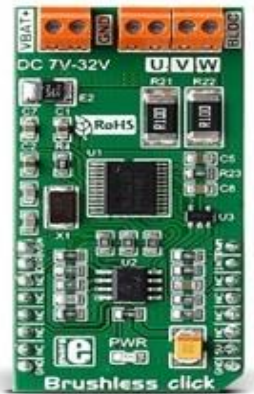

(c)

Figure 1. A photo-image of the (a) Brushless motor shows rotor, shaft, and coils, (b) Microchip PIC18F47J53 microcontroller, (c) Brushless click

To ensure the stability of spin speed, a feedback control technique was designed, simulated and employed. The purpose of the feedback control circuit is to compare the desired speed with the measured speed. An error is recognized when there is a difference between these two values. The error translated through a proportional-integral-derivative (PID) controlling algorithm to decide the required acceleration or damping that important for spinning speed integrity [17-19]. The feedback control circuit alongside the USB communication is designed and simulated using the Proteus simulation system (Design Suite by Labcenter Electronic "version 8.5"). The Proteus simulation is used to operate and test the electronic circuitry before it is actually implemented. The speed sensor was replaced with a pulse generator and the motor control circuit replaced by an oscilloscope. Figure 2 illustrates the simulation of the feedback control circuit design by Proteus system.

To operate the system, the user has to enter particular parameters (Speed and Interval) and to translate that in the MCU, a combination of timer-counter scheduling value has been used. In the microcontroller two timer modules used to synchronize system timing and accomplish user setup. Timer 0 and timer 3 have been carefully chosen to operate the system tasks that related to any time scheduling tasks required. Figure 3 shows the flowchart of the timers running sequence to calculate the spinning speed and intervals. As shown in the chart, timer 0 uses to count the user interval by creating the timer interrupt each 1 second and count these seconds until approach the destination value of user interval in seconds. During this 1 second calculation, timer 3 uses to count the number of pulses each 1 second. These pulses represent the number of cycles that come from the IR module (rpm).

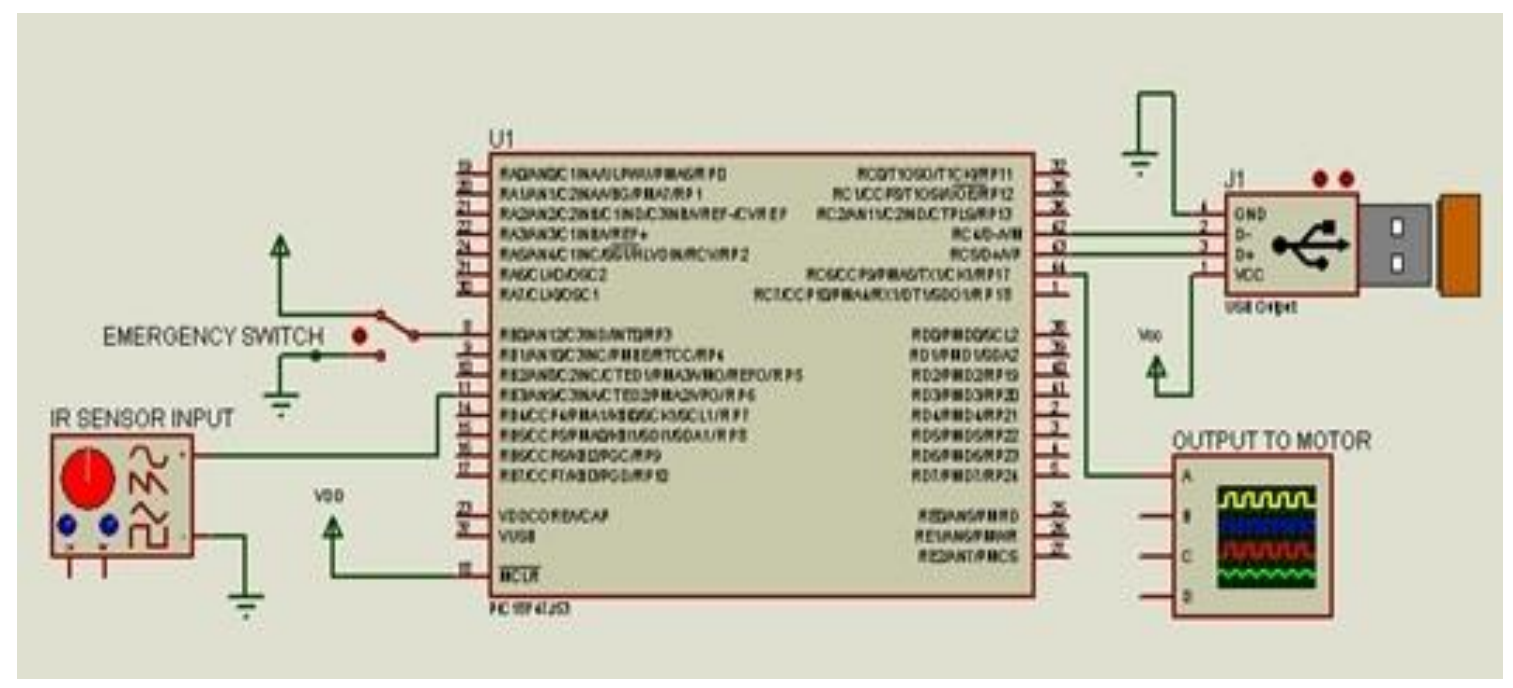

Figure 2. Simulation of the feedback control circuit design by proteus system 


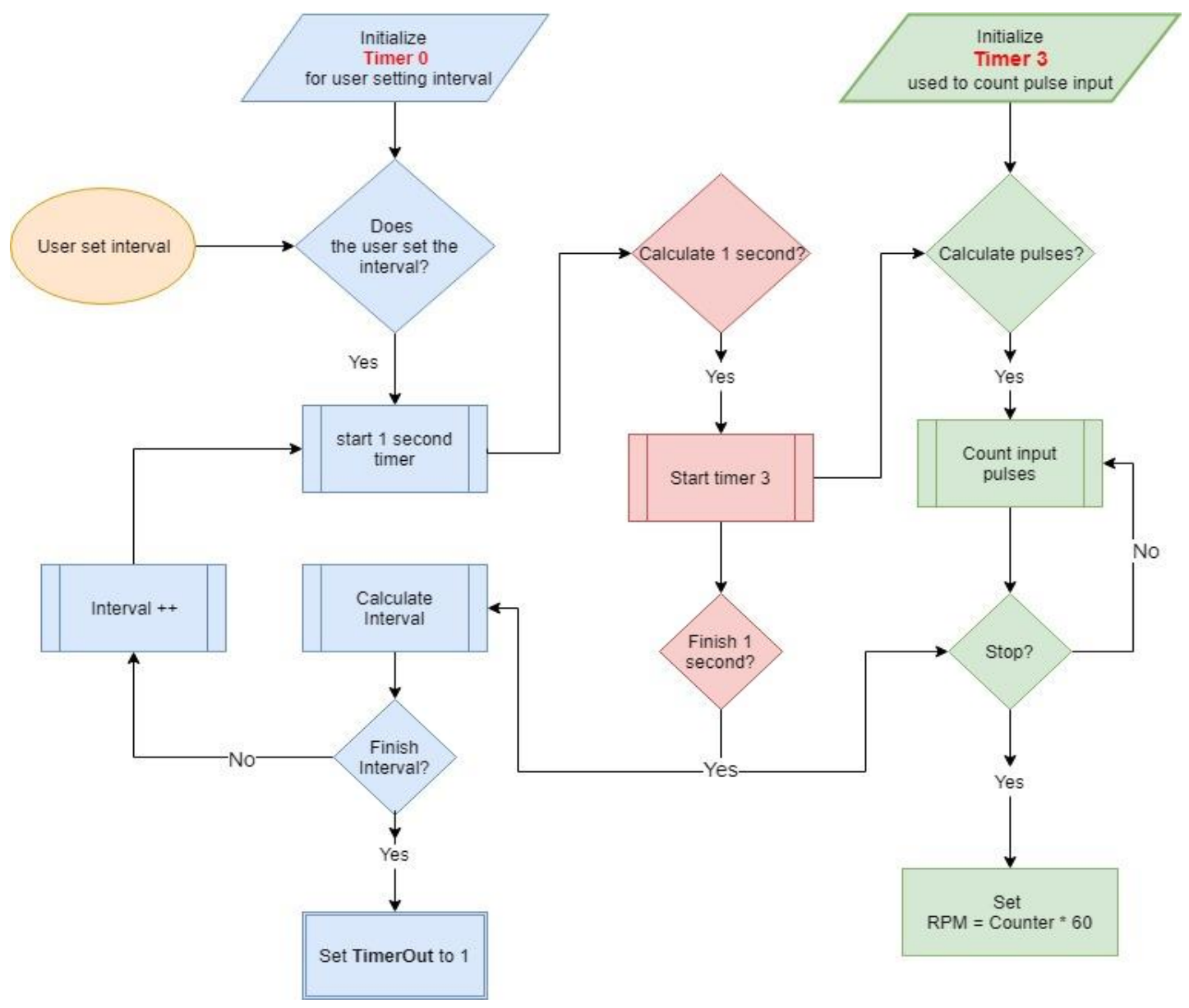

Figure 3. Timers running sequence flowchart

Users use a computer application user interface to enter test parameters. Tests can be done by two modes of operation: standard and manual mode. In the standard mode particular sets of the sequence have been stored and ready to use directly, while in manual mode the user free to select the task parameters whether it is a single-step or multi-step task, and what is the speed and interval for each step. By pressing the run button a connection between the computer and the device established, and user test parameter transferred to the device inside the MCU. The MCU creates combinations of timer-counter settings and lets the timer modules execute them. For example, to apply two multi-step spinning speed, the interaction box allows the user to enter two types of spinning tasks. The tasks will execute alternately, first speed and interval and then the second speed and interval. The feedback control mechanism utilized by the number of pulses generated from the Infrared (IR) sensors and faded back to the microcontroller through a calibration circuit [20]. IR speed sensor is used to measure the spinning speed without affecting the speed of the motor. The working principle of the sensor can be summarized by, the IR LED transmitter sending continuously the infrared waves to the moving part of the engine and receive the reflective signals via the IR photodiode receiver continuously. A selected portion of moving part of the motor dye with black color, because the black dye absorbs the largest amount of infrared radiation (low value of reflectance). As a result, the signal generated by the photodiode receiver is a pulse in the number of rotation cycles. Within the PIC18F47J53 microprocessor, a function is created to calculate the pulses generated by the signal received from an IR photodiode per second.

\subsection{Spinning Speed Calculations}

In order to achieve a wide range and a high resolution of the operation speed of spin coater apparatus, three different voltage stages have been implemented 5, 7 and $10 \mathrm{~V}$. The three voltage stages implementation extracted based on experimental measurements. It is found to be the three stages technique gives a smoother and wider range of spinning speed than single voltage stage. According to the extracted data, a special driving circuit is developed to control the motor speed of the studied spin coater apparatus. 
The spin coater apparatus can run at the desired rotational velocity by mean of maximum speed and control the PWM duty cycle. However, the experiment shows that the supply voltage level can shift the spinning rang. The spin coater system performance has examined experimentally before final setting to extract the expression to relate spinning speed, duty cycle and supplied voltage. It is found to be that, each supply voltage stage, gives a specific range of running speed of the spin coater during the variation of the duty cycle of PWM. Figure 4 illustrates the manner of actual spinning speed changing with supply voltage range from 5 to $10 \mathrm{~V}$ and the entire duty cycle of the PWM signal as the 3 dimensional surface. The fit tools with linear interpolation are used to plot a 3 dimensional surface in MATLAB package. It is clearly seen from Figure 4 that the BLCD motor behave differently with different supplied voltages. The duty cycle versus spinning speed plan shows that, for all studied voltages, the speed rises with increasing the duty cycle until reach a particular value of $\mathrm{D}$, then after this particular value the velocity tends to saturate. For example, for $5 \mathrm{~V}$ input level, when the $\mathrm{D}$ equal to $35 \%$ the velocity is $1500 \mathrm{rpm}$, by increase the D the speed increases accordingly, however, the velocity statured at $3840 \mathrm{rpm}$ when the duty cycle above $68 \%$ up to $100 \%$. Hence, the spinning speed velocity range is 1500 to $3840 \mathrm{rpm}$ for $5 \mathrm{~V}$ applied voltage level, which is much lesser than the speed range capability of BLCD. For $7 \mathrm{~V}$ applied voltage the speed is $2640 \mathrm{rpm}$ for $35 \% \mathrm{D}$, the spinning increases with increases the duty cycle, the speed started to be saturated $5460 \mathrm{rpm}$ at $64 \%$ up to $100 \%$ D. Similarly, for $10 \mathrm{~V}$ stage, the speed is $4380 \mathrm{rpm}$ for $35 \% \mathrm{D}$, the speed started to be saturated $7920 \mathrm{rpm}$ at $60 \%$ up to $100 \% \mathrm{D}$. Accordingly, three platforms technique deployed to the proposed system to get the full range 1500 to $7920 \mathrm{rpm}$. Table 2 summarizes the three stages of performance. Consistently, based on the extracted data, the PID controller was designed to control the duty of PWM signal of the dc motor to ensure that the error between desired and actual running velocity has the minimum value.

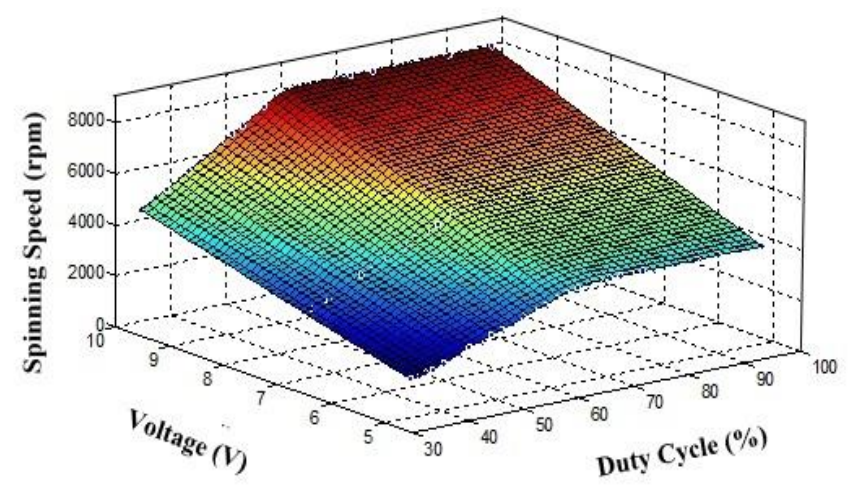

Table 2. The Three Stages of Performance

\begin{tabular}{cccc}
\hline $\begin{array}{c}\text { Input } \\
\text { Voltage } \\
\begin{array}{c}\text { Stage } \\
(\mathrm{V})\end{array}\end{array}$ & $\begin{array}{c}\text { Initial } \\
\text { Velocity } \\
(\mathrm{rpm}) @\end{array}$ & $\begin{array}{c}\text { Saturated } \\
\text { Velocity } \\
(\mathrm{rpm})\end{array}$ & $\begin{array}{c}\text { D (\%) @ } \\
\text { Saturated } \\
\text { Velocity }\end{array}$ \\
\hline 5 & 1500 & 3840 & 68 \\
7 & 2640 & 5460 & 64 \\
10 & 4380 & 7920 & 60 \\
\hline
\end{tabular}

Figure 4. The measured spinning speed changing with supply voltage and duty cycle of PWM signal as 3 dimensional surface

\subsection{Connect the System to the Computer}

In order to connect the system to the computer, a serial transfer technology was used via the USB port. Human interface device (HID) protocol is a technology allows the external devices to be connected to the computer by supported bi-directional communication. The PIC18F47J53 microprocessor has the ability to output data to the computer via USB HID protocol and vice versa. HID has an efficient working inbuilt library and does not require any additional library or devices. The system is developed and programmed by using MikroC compiler from Mikroelectronika using USB HID library and USB descriptor tool.

\subsection{Spin Coating System Construction}

The circuit and the motor were fixed in a wood box of 20x20x10 $\mathrm{cm}^{3}$ with the axis of the motor passing through the spill container. A Teflon (Multi-tetrafluoroethylene) spill container has been fabricated to get the desired spinning stage in order to reduce material splashing and wasting. The spill compartment Teflon cover with a heavy glass window slot to allow visibility and safety during the coating process. Teflon has been chosen because of its unique properties. It is a durable, available and chemically inert material that does not react with acids, bases and organic solvents. Figure 5 shows the spin coating system photographs. 

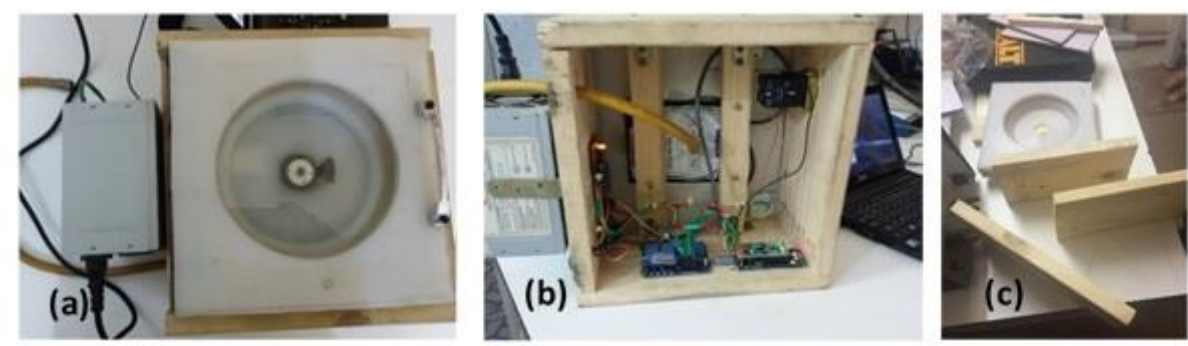

Figure 5. A photo-image of the (a) The spin coating system, (b) Backside of the spin coater where the electronics circuit is fixed, (c) Spin coater while construction

\section{RESULTS AND DISCUSSION}

In order to evaluate the proposed spin coater performance, the system is used to deposit $\mathrm{ZnO}$ thin films on the glass substrates by sol-gel method. The $\mathrm{ZnO}$ sol-gel is prepared as follows; Zinc acetate dihydrate $\left(\mathrm{Zn}\left(\mathrm{CH}_{3} \mathrm{COO}\right)_{2} .2 \mathrm{H}_{2} \mathrm{O}: \mathrm{ZnAc}_{2} .2 \mathrm{H}_{2} \mathrm{O}\right)(0.5 \mathrm{M})$ was dissolved in a solution consisting of $20 \mathrm{~mL}$ isopropanol $\left(\left(\mathrm{CH}_{3}\right)_{2} \mathrm{CHOH}\right)$ and $1 \mathrm{~g}$ diethanolamine $\left(\right.$ DEA: $\left.\left[\mathrm{CH}_{2}(\mathrm{OH}) \mathrm{CH}_{2}\right]_{2} \mathrm{NH}\right)$ at room temperature $(300 \mathrm{~K})$. The molar ratio of zinc acetate to DEA was 1:1. At room temperature, the solution was stirred for 1 $\mathrm{h}$ to get a clear and transparent solution that was used for spin coating. [21]

To obtain a thin film on a glass substrate by a spin coater, the substrate must be fully dry and completely clean. The $2.5 \times 2.5 \mathrm{~cm}^{2}$ glass substrates were cleaned before deposition. The cleaning process was carried out on the following steps; the substrates were soaked in the isopropanol sonicated for 2 minutes, immersed in the acetone for 1 minute, rinse and washed with deionized water, then dried. [22]

\subsection{Spin Coater in Use}

Two groups of samples are used to determine the coating quality, each group of samples has a different set of coating parameters. Spin speed parameter varying from 2000-7000 rpm for the spin time equal to 40 seconds, that for the first group. For the second group, the spin time parameter was changed from 10 to 90 seconds at $4000 \mathrm{rpm}$. The volume of solution used for each sample is $0.25 \mathrm{ml}$. All coatings were followed by pre-heating the at $373 \mathrm{~K}$ for $10 \mathrm{~min}$ and post-heating at $723 \mathrm{~K}$ for $1 \mathrm{~h}$ in an air-ambient muffle furnace for dried and calcined, respectively. [23] The thickness of each sample was measured using spectroscopic reflectometer system type thickness film probe (TFProbe) from Angstrom Advance Inc.

Figure 6 shows spinning speed versus $\mathrm{ZnO}$ thin film thickness coated with the built system. The spinning speed within the range of $2000-7000 \mathrm{rpm}$, spin time by is equal to 40 seconds. The figure illustrates that the thickness of the membrane decreases with the increase of the spinning speed of the device. The illustrated results show an agreement with previously reported results. [24] At $2000 \mathrm{rpm}$, the thickness of the thin film is about $400 \mathrm{~nm}$ and further increases to $4000 \mathrm{rpm}$, the thickness reduced to the half $(200 \mathrm{~nm})$. When the speed increases to $7000 \mathrm{rpm}$, the thickness becomes about $115 \mathrm{~nm}$. As the speed increases, the rate of the change in thickness is reduced.

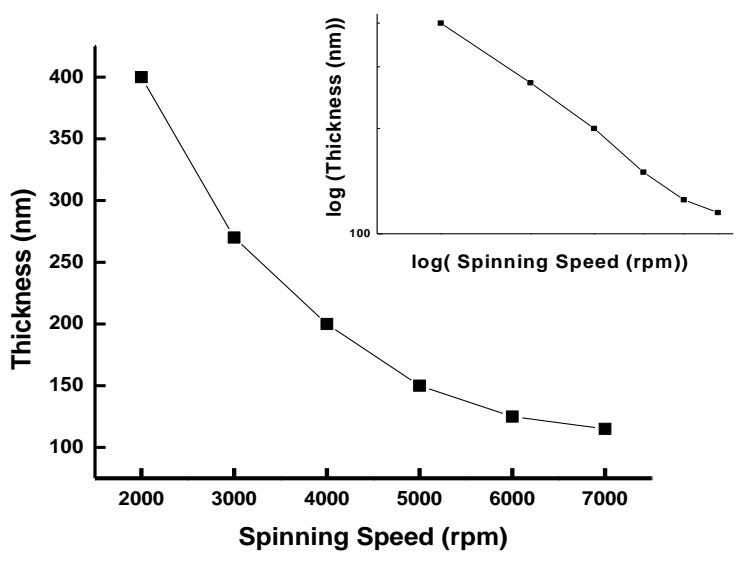

Figure 6. Effect of the spinning speed on $\mathrm{ZnO}$ thin film thickness

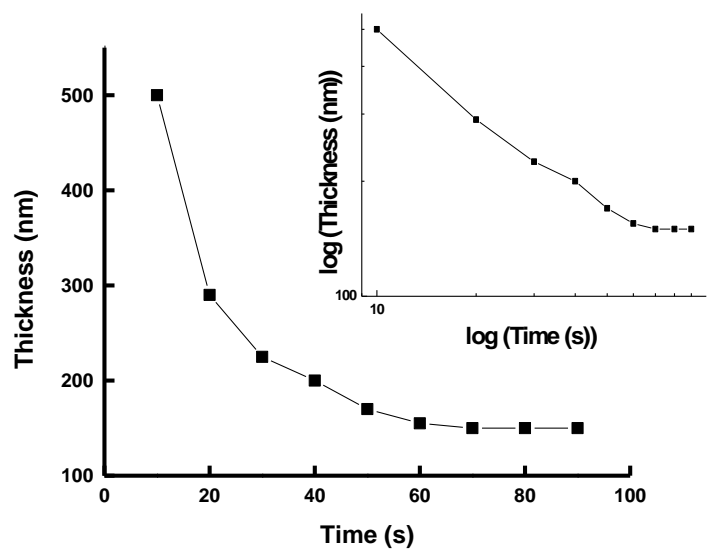

Figure 7. Effect of the spinning speed time on $\mathrm{ZnO}$ thin film thickness 
It is well known that, the thin film thickness proportion with the evaporation rate of the solvents. There are three possible cases that determine the thickness of thin films:[10] (A) When the solvent does not evaporate, the thickness of the thin film $(h)$ is proportional to the spinning speed $(\omega)$ and the spinning time $(\mathrm{t})$ ( $\mathrm{h} \alpha \omega^{-1} \mathrm{t}^{-1 / 2}$ ), (B) When the solvent evaporates at a constant evaporation rate, the thickness of the thin film is proportional to the speed of rotation $\left(\mathrm{h} \alpha \omega^{-2 / 3}\right)$, (C) When the solvent evaporates at not-constant evaporation rate, the thickness of the thin film is proportional to the square root of spinning speed $\left(\mathrm{h} \alpha \omega^{-1 / 2}\right)$.

Figure 6 demonstrates that the thickness of the membrane decreases with the increases of the spinning speed. From nonlinear fit, by using Origin software version 6 , it is found to be the thickness proportional with $\omega^{-1}$ and follows a case (A) above, in such case $(h)$ is proportional to the spinning speed $\omega^{-1}$ and the spinning time t. The inset of Figure 6, shows the log-log data of Figure 6 to clarify dependencies on $\omega^{-1}$. Figure 7 illustrates the spinning time versus the average thickness at a particular speed of $4000 \mathrm{rpm}$. At spinning time equal to 40 seconds, the average thin film thickness is $200 \mathrm{~nm}$. When the time is increased to 60 seconds, the film thickness becomes 155 nanometers, further increased to 90 seconds, the thickness becomes $150 \mathrm{~nm}$ and the thickness decreases-rate gradually reduces and reaches a specific value. This phenomenon can be due to the viscosity change of the $\mathrm{ZnO}$ gel-sol during the coating process. From the nonlinear fit of the Figure 7 curve, it is found to be the thickness proportional with $\mathrm{t}^{-0.6}$, which is very close to the case (A) that thickness proportion to $\mathrm{t}^{-0.5}$. The inset of Figure 7, shows the log-log data of Figure 7 to clarify dependencies on $\mathrm{t}^{-0.6}$. These results are consistent with the previously reported work to deposit thin films obtained by a sol-gel method using commercial coating devices [25].

\section{CONCLUSION}

We design, implement and characterize a feedback programmable spin coater. This system is built from a commercially available hard drive DC motor, vacuum pump, IR feedback circuit, brushless click carries, microcontroller. In addition, the system is connected to the computer through an interactive user interface designed with the programming languages (Visual Studio.Net). The main feature of this system a wide range of stable accurate multi-step spinning speeds using feedback circuits and PWM technique to overcome the drawbacks of the traditional systems such as speed degradation with utilization. Three different voltage stages have been implemented 5,7 and $10 \mathrm{~V}$ to achieve a wide range and high resolution of the operation speed of spin coater apparatus. $\mathrm{ZnO}$ thin films deposited by sol-gel technique on glass substrates produced with the built system. The resulting thin films are uniform and homogenous with accurate ability to control their thickness by high precision controlling of spinning speed and time. As a result, the proposed system is a low-cost, high-efficiency and durable system since the system efficient daily use in our laboratory for about 1 year.

\section{REFERENCES}

[1] Z. Ye, L. Lu, and M. Wong, "Zinc-oxide thin-film transistor with self-aligned source/drain regions doped with implanted boron for enhanced thermal stability," IEEE Trans. Electron Devices, vol. 59, no. 2, pp. 393-399, 2012.

[2] D. B. Potter, M. J. Powell, I. P. Parkin, and C. J. Carmalt, "Aluminium/gallium, indium/gallium, and aluminium/indium co-doped $\mathrm{ZnO}$ thin films deposited: Via aerosol assisted CVD," J. Mater. Chem. C, vol. 6, no. 3, pp. 588-597, 2018.

[3] S. Singh, G. M. Ali, and P. Chakrabarti, "Fabrication and characterization of $\mathrm{ZnO}$ thin films prepared by thermal oxidation of vacuum deposited Zn," Sci. Adv. Mater., vol. 3, no. 6, 2011.

[4] N. Y. Mostafa, A. Badawi, and S. I. Ahmed, "Influence of $\mathrm{Cu}$ and $\mathrm{Ag}$ doping on structure and optical properties of In2O3thin film prepared by spray pyrolysis," Results Phys., vol. 10, no. May, pp. 126-131, 2018.

[5] K. Deva Arun Kumar, V. Ganesh, M. Shkir, S. AlFaify, and S. Valanarasu, "Effect of different solvents on the key structural, optical and electronic properties of sol-gel dip coated AZO nanostructured thin films for optoelectronic applications," J. Mater. Sci. Mater. Electron., vol. 29, no. 2, pp. 887-897, 2018.

[6] A. S. Ismail, M. H. Mamat, M. F. Malek, M. M. Yusoff, N. D. Sin, and S. S. Shariffudin, "Intrinsic ZnO / Al-doped ZnO Homojunction : Structural and Optical Properties," vol. 12, no. 1, pp. 393-398, 2018.

[7] F. Bittner, T. Oekermann, and M. Wark, "Scale-up of the electrodeposition of ZnO/Eosin Y hybrid thin films for the fabrication of flexible dye-sensitized solar cell modules," Materials (Basel)., vol. 11, no. 2, 2018.

[8] G. M. Ali, "Interdigitated Extended Gate Field Effect Transistor Without Reference Electrode," J. Electron. Mater., vol. 46, no. 2, pp. 713-717, 2017.

[9] M. Shibata, Y. Sakai, and D. Yokoyama, "Advantages and disadvantages of vacuum-deposited and spin-coated amorphous organic semiconductor films for organic light-emitting diodes," J. Mater. Chem. C, vol. 3, no. 42, pp. 11178-11191, 2015.

[10] R. F. Bianchi, M. F. Panssiera, J. P. H. Lima, L. Yagura, A. M. Andrade, and R. M. Faria, "Spin coater based on brushless dc motor of hard disk drivers," Prog. Org. Coatings, vol. 57, no. 1, pp. 33-36, 2006.

[11] N. Sahu, B. Parija, and S. Panigrahi, "Fundamental understanding and modeling of spin coating process: 
A review," Indian J. Phys, vol. 83, no. 4, pp. 493-502, 2009.

[12] A. Gaur and D. Rana, "Development of Spin Coating System Based on AC Universal Motor for Deposition of Polymer Films," J. Sensors Instrum., vol. 2, no. 1, pp. 1-8, 2014.

[13] N. Manikandan, B. Shanthi, and S. Muruganand, "Construction of Spin Coating Machine Controlled by Arm Processor for Physical Studies of PVA,” Int. J. Electron. Electr. Eng., vol. 3, no. 4, pp. 318-322, 2014.

[14] I. Pratama, J. Y. Mindara, D. W. Maulana, C. Panatarani, and I. M. Joni, "Development of spin coater with close loop control system using ATMega8535 microcontroller," AIP Conf. Proc., vol. 1712, pp. 1-6, 2016.

[15] B. A. Obaid, A. L. Saleh, and A. K. Kadhim, "Resolving of optimal fractional PID controller for DC motor drive based on anti-windup by invasive weed optimization technique," vol. 15, no. 1, pp. 95-103, 2019.

[16] P. Yadav, S. Khan, Y. Chauhan, and S. Chauhan, "Speed Control of DC Motor USING," Int. J. Res., vol. 1, no. 10, pp. 1719-1724, 2014.

[17] M. A. A. Mohamed, A. M. Elmahalawy, and H. M. Harb, "Developing an Open Architecture and Intelligent System for Speed and Direction Controlling (PC-SDC) of DC Motors," Arab. J. Sci. Eng., vol. 39, no. 12, pp. 8793-8810, 2014.

[18] S. N. Al-bargothi, G. M. Qaryouti, and Q. M. Jaber, "Speed control of DC motor using conventional and adaptive PID controllers," vol. 16, no. 3, pp. 1221-1228, 2019.

[19] P. T. Tin, L. A. Vu, M. Tran, N. Q. Dung, and T. T. Trang, "Optimal tuning pid controller of unstable fractional order system by desired transient characteristics using RIM," vol. 14, no. 3, pp. 1177-1188, 2019.

[20] A. A. New, A. A. Zan, and W. P. Aung, "Control System Consideration of IR Sensors based Tricycle Drive Wheeled Mobile Robot,” Int. J., vol. 88704, pp. 30-36, 2008.

[21] G. M. Ali and P. Chakrabarti, "Performance of $\mathrm{ZnO}$-based ultraviolet photodetectors under varying thermal treatment," IEEE Photonics J., vol. 2, no. 5, 2010.

[22] S. Sharma, S. Vyas, C. Periasamy, and P. Chakrabarti, "Structural and optical characterization of ZnO thin films for optoelectronic device applications by RF sputtering technique," Superlattices Microstruct., vol. 75, pp. 378-389, 2014.

[23] G. M. Ali and P. Chakrabarti, "Effect of thermal treatment on the performance of ZnO based metal-insulatorsemiconductor ultraviolet photodetectors," Appl. Phys. Lett., vol. 97, no. 3, 2010.

[24] A. L. Bogdanov, "Use of SU-8 negative photoresist for optical mask manufacturing Use of SU-8 Negative Photoresist for Optical Mask Manufacturing," no. June 2000, 2015.

[25] H. K. Lee, S. Il Chang, and E. Yoon, "A flexible polymer tactile sensor: Fabrication and modular expandability for large area deployment,” J. Microelectromechanical Syst., vol. 15, no. 6, pp. 1681-1686, 2006.

\section{BIOGRAPHIES OF AUTHORS}

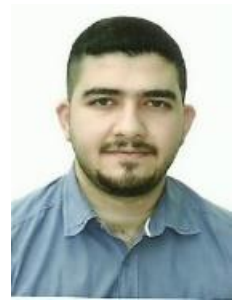

Ahmed Khalid Kadhim was born in Baghdad, Iraq, in 1987. He received the B.E. degree in electrical engineering from the University of Mustansiriyah, Baghdad, Iraq, in 2009, and the M.Sc. degrees in microelectronic engineering from the Newcastle University, UK, in 2016. In 2012, he joined the Department of Electrical Engineering, University of Mustansiriyah, as a demonstrator in the electronic laboratory, and in 2016 became a Lecturer. His current research interests include $\mu$ systems, embedded systems, and processors, internet of things (IoT), cloud computing, sensors, robotics design.

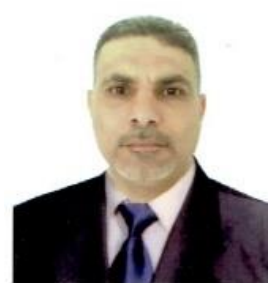

Salah M. Swadi was bornin1973. He has finished his BSc degree in Mechanical Engineering from the University of Technology, Baghdad in 1999 and MSc degree in Mechanical Engineering from the University of Technology in 2002. He was appointed as an Assistant Lecturer in Automated Manufacturing Engineering Department/ University of Baghdad in 2006. He has finished his Ph.D. degree in Mechanical Engineering from the University of Technology, Baghdad in 2016. His present research interests concern dynamics of mobile robotics design and control, and embedded systems.

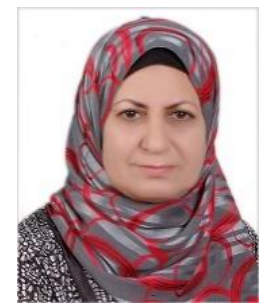

Ghusoon M. Ali received the B.Sc. degree and MSc in Electrical Engineering from the University of Techology, Baghdad, Iraq, in 1984 and 1989, respectively. The Ph.D. degree from the Institute of Technology, IIT Varanasi, Varanasi, India, in 2011. She joined the Electrical Engineering Department, College of Engineering, Mustansiryah University, Baghdad, Iraq, in 1990, as an Asst. Lecturer, where she has been an Asst. Professor since 2016. Her current research interests include the thin-film based electronic and optoelectronic devices. 